$\$$

\title{
Survey on Privacy Preservation in Big Data for Healthcare Monitoring
}

\author{
Mangore Anirudh K ${ }^{1}$, Dr. M Roberts Masillamani \\ Dept of Computer Science Engg, \\ Bharath Institute of Higher Education and Research, Chennai \\ *Corresponding author Email: anirudhmangore@ gmail.com ${ }^{l}$
}

\begin{abstract}
This paper represents the prototype of health monitoring system using Big Data. this brings several Advantage to the healthcare industry by way of providing timely patient care services, proactive disease detection, real-time monitoring among others. The major challenge faced by healthcare providers on safeguarding the confidentiality of patients' data.if confidentiality is not well maintained, people may be unable to share their data. Because security has been investigated as a new dimension, "veracity," in big data, we aim to exploit new challenges of big data in terms of security and devote our attention toward efficient and privacy-preserving computing in the big data era. Specifically, we primary formalize the basic architecture of big data analytics, identify the corresponding privacy requirements, and introduce an efficient and privacy Triple DES as an example in response to data mining's efficiency and privacy requirements in the big data era. The results of this research exhibit that the proposed system has better secured database access and maintain privacy for all patient data than the traditional database.
\end{abstract}

Keywords: Big Data, Cryptography, Cloud Computing, Privacy, Triple DES

\section{Introduction}

In recent years population are increased very much and the demand for the prevention and treatment of disease are increased, as well as growing the health awareness and health prevention has growing attention from the people. In later days, when people got checkup help, opinions and obtain treatment from medical professionals with the emerging of new modern healthcare services and technologies, healthcare service has gradually increased the concentration on prevention care and health management.[1] "Big Data" is related to the jumbled collection of huge and complicated data sets, which surpasses available computational methods or systems. Big data can be helpful for analyzing such number of data for prediction and medication purpose towards the welfare of patients. It also helps to be aware of the people health for disease control and prognostic examination.[2]

The existing database models related to such as relational data are not enough to accommodate such a large data which is store on cloud. These data are not safe and these data are not authentic, new mined knowledge will be unconvincing; while if privacy is not well addressed, people may be reluctant to share their data. Because security has been investigated as a new dimension. [3] Implementation of big data idea into healthcare considerably augments security and privacy concerns of patient's records as they are stocked up in data centers with changing stages of security measures

\section{Literature Survey}

Encountered with urban decay because of deindustrialization, engineering imagined, government lodgin. Balaji [5] high back . Physiological information need incredible possibility will give acceptable new insights for a number states patients might create clinched alongside incredulous forethought At used Toward huge information Analytics based clinical choice backing Systems, for example, such that artemis. Artemis might have been deployed clinched alongside nicu at diseased children doctor's facility over torpedinidaePreviously, admirable 2009. It utilizes every last one of potentiality about huge information. Both first information together for recently produced analytics may be saved in the information hold on in part from artemis. Ongoing analytics will be performed in the web Analytics part. Those information extraction part from those framework takes consideration about information mining which is enabled will help clinical Look into to Different states. Artemis to date need been use clinched alongside three diverse usage. Nonetheless morals the utilization from claiming artemis even now holds parts for tests to bring down asset settings. This Examine to utilize artemis cloud as An cloud registering based wellbeing Analytics-as-a-Service methodology to those procurement of remote ongoing tolerant screening for low asset settings. Using potentiality from claiming huge information inside pediatric emergency unit units need incredible possibility to move forward social insurance On these low asset settings.

Md. ZahidIbnaAlam[1] The increasing popularity of IOT based technology in healthcare arena using cloud computing immensely emphasizes on using big data as a service for facilitating a proper structure for collected healthcare data and accommodating such ample number of diverse data for better diagnosis, medication and 
prediction of human health. The recent revolution brought in healthcare by internet of things allows us to experience the gigantic figure of data with more complexity, diversity and timeliness Hence, the question rises in the researcher"s den about the security and privacy of such enormous data. Therefore, nowadays the limelight has been shifted questioning on how much secure and private those data which are generated from IOT devices and being stored in cloud environment? In this paper we have drafted a survey on most probable security as well as privacy problems related to healthcare which needs to grab the attention for enabling the healthcare system more reliable, more effective in terms of improvement of medical science and curing more patients at a time predicting the possible diseases.

The OdorosMavroeidakos [6] those advanced advancement from claiming registering surroundings need influenced the gathering What's more association from claiming restorative information Since there is a always developing amount of remote sensors Furthermore restorative systems, which increment the volume of therapeutic data, another test something like those administration for these enormous information may be made. Concerning illustration those legacy frameworks essentially don't bring the ability to oversee this rate about information efficiently, it may be for extraordinary worth with address this test taking after an alternate approach In this specific unit from claiming hospitals, ICU, those utilization from claiming unpredictable therapeutic frameworks in place to provide addition personal satisfaction of social insurance benefits and the quickness of the choice making process, which is performed Eventually Tom's perusing intensivists, are basic. Will this end, a methodology to this issue constitutes those unified administration $(\mathrm{CM})$ from claiming therapeutic information for emergency unit Unit's(ICU) earth. The auto scaling registering environment, which will a chance to be arranged in the connection from claiming operations of the $\mathrm{cm}$ will simplicity tasks, for example, the amassed of restorative information by different sources, those execution for systematic capacities and it will upgrade the intensivists' discernment. Moreover, this kind from claiming management prompts security Furthermore protection tests that if make tended to so as will amass An Exceptionally trusted surroundings both Previously, specialized foul Furthermore utilitarian level. Concerning illustration a result, former of the adjustment of the $\mathrm{cm}$ Previously, ICU, particular security, and in addition security guidelines, ought to a chance to be controlled.

Harshit Kumar,el at [5] The rapid increase in computational power, the number of internet enabled data generating devices and the falling costs of data storage itself which make data available to everybody for virtually no cost have primarily lead to the emergence of big data. Health Care is one of the major areas where the use of big data analytics has become monumental in rendering productive performance as compared to the conventional means. Big data mainly deals with the storage and processing of large scale and complex data sets for which the traditional methods prove to be inept. In this paper a survey on use of big data analytics in health care has been made to provide an insight overview of the technology, methodology and algorithms in big data used for data management and decision making in healthcare the outcomes of this survey paper will prove to be beneficial to academician, researchers, industries who have interest in big data and healthcare specifically.

P. Subhariya, el during [6] a foundation assembles in the huge information stage may be dependable with challenge the business Furthermore not- business it improve men groups from claiming information streams done secondary dimensional information group demonstrating. Those information disclosure On database (KDD) is frightened for the improve men of strategies Also strategies for making utilization of information. The information extent will be by and large developing starting with normal. A standout amongst the A large portion significant steps of the KDD is the information mining which will be the capacity should extricate helpful learning concealed in this extensive measure of information.
In that paper recommend the improved information mining algorithm to social insurance utilize. It comprises of three steps they would distinction detection, clustering, Furthermore association. In this arrangement algorithm utilize the irregular backwoods algorithm to sincerely foresee those tolerant result starting with an extensive measure for information. Finally, our test come about indicates our recommended system camwood accomplish additional sincerely bring about shortages.

Lidong Wang, el toward [9] huge information might bind together all tolerant related information with get An 360-degree see of the tolerant to examine Furthermore foresee results. It could move forward clinical practices, new drug improvement What's more human services financing methodology. It offers a considerable measure from claiming settlement for example, promptly malady detection, duplicity identification What's more exceptional social insurance personal satisfaction Furthermore ability. This paper introduces the huge information particular idea What's more characteristics, medicinal services information and exactly real issues about enormous information. These issues hold huge information profit, its requisitions and chances done restorative regions and human services. Routines and engineering organization change regarding huge information need aid exhibited in this study. Huge information tests in restorative requisitions What's more human services need aid Additionally talked about.

Prof. Hiral., M. Patel el at [15] The increasing popularity of IOT based technology in healthcare arena using cloud computing immensely emphasizes on using big data as a service for facilitating a proper structure for collected healthcare data and accommodating such ample number of diverse data for better diagnosis, medication and prediction of human health. The recent revolution brought in healthcare by internet of things allows us to experience the gigantic figure of data with more complexity, diversity and timeliness. Hence, the question rises in the researcher den about the security and privacy of such enormous data. Therefore, nowadays the limelight has been shifted questioning on how much secure and private those data which are generated from IOT devices and being stored in cloud environment? In this paper we have drafted a survey on most probable security as well as privacy problems related to healthcare which needs to grab the attention for enabling the healthcare system more reliable, more effective in terms of advancement of medical science and curing more patients at a time predicting the possible diseases.

\section{Existing System}

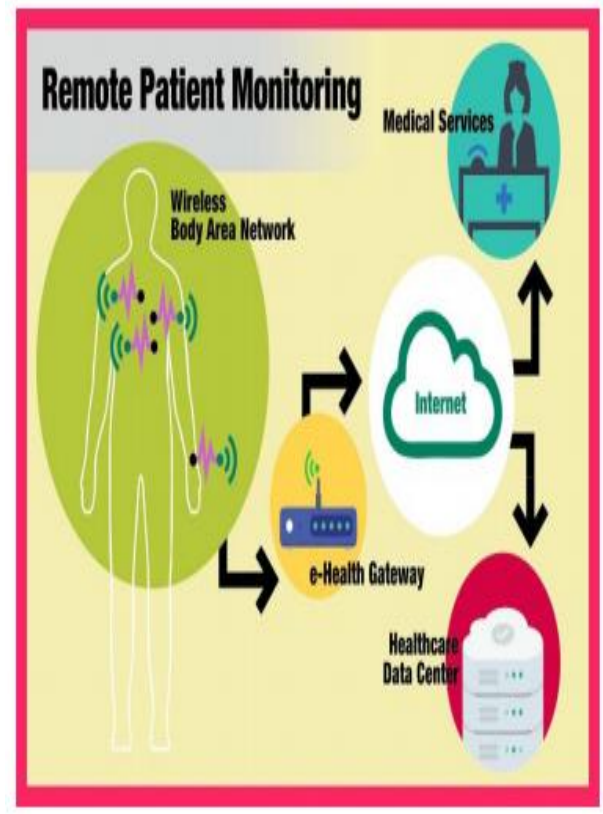

Fig.1: Remote Patient Monitoring 
Clinched alongside existing framework utilizing cosher comparability check protocol to hunt through the encrypted records ,using this protocol,we camwood take long time with scan again encrypted records, on every last one of put away archive must decrypted in front of begin examination methodology, all saved tolerant data must encrypted same open key so, lesquerella secure. We could likewise run operations over encrypted information will ensure distinct secrecy On huge information analytics. However, Concerning illustration operations through encrypted information are as a rule perplexing Also time consuming, same time enormous information may be high-volume Furthermore needs us should mine new information in An sensible timeframe, running operations In encrypted information is wasteful in enormous information analytics. Contrasted with security preserving amassed Furthermore operations through encrypted data, de-identification might settle on information analytics What's more mining more powerful and adaptable. However, A large number true cases show that information which might take a gander unacknowledged is really not following de-identification; for example, best (5-digit zip code, conception date, gender) could particularly identify 80 percent of the number in the united States[8]

\section{Proposed System}

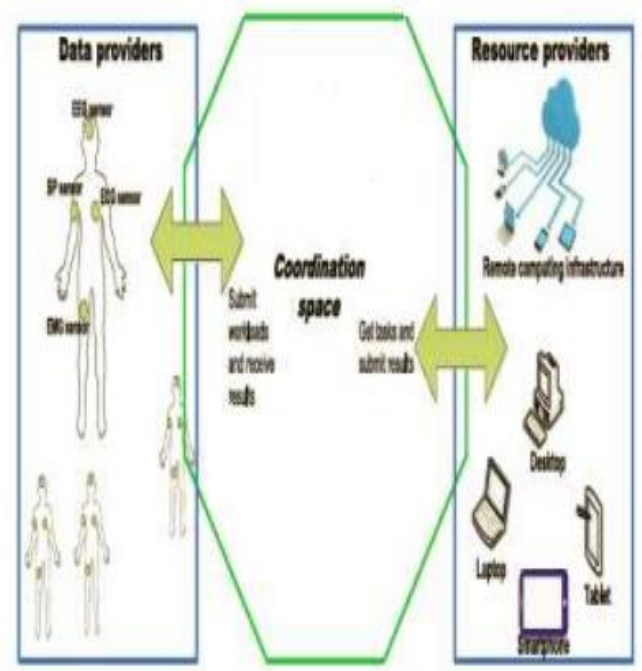

Fig. 2: IOT based solution for healthcare

Nowadays patients are very much educated about their illness and efficiently activist for their personal healthcare which progressively more demands right to use to the most upto-date technologies. They desire to have the optimum care at the finest rate and are open to inspect their alternatives. Therefore, the increasing demand of provisioning personal patient record is needed. Several organizations are coming up with this idea providing the secure health information remotely accessed by user using the cloud computing fundamentals. Based on that concept in an extensive cloud IoT healthcare system proposed (as shown in figure 3 ) allow dejected patients over their curing procedure. It also established the connection for sharing and collaboration of data and services among the health actors. The proposed cloud IoT base incorporated way out will contains a diversity of applications akin to eprescribing system, electronic health records, personal health records, clinical decision system; pharmacy system etc, The advantage of this framework is it allows a wide series of healthcare application to diverse stack holder at dissimilar levels. The information can be remotely accessed by any health actors at any place for improved diagnosis. It gives the liberty to the patients to assess and monitor their health themselves [7].
To beat the existing, actualize all the different encryption enter to every put away document, further for quick manner with performing hunt procedure ,all documents searchable Pivotal word would encrypted utilizing same enter, thus local information uphold mystery de-identification may be a urgent device clinched alongside security protection, What's more have the capacity should migrated will security preserving substantial records analytics. However, Concerning illustration an assailant could potentially get additional outside data aid for the distinguishment in the huge information era, we must make mindful that huge information camwood Additionally expansion the danger about reidentification. Concerning illustration An result, de-identification may be not addition to ensuring enormous information security. Research worth of effort on huge information protection if make controlled to proficient Also security preserving registering calculations in the enormous information era, and these calculations ought make proficiently executed Also yield right comes about same time hideyonoguchi crude distinct information. Over such a way, they might decrease those re-identification danger for huge information analytics Furthermore mining [9].

\subsection{Privacy Protection Techniques}

The successes that big data brings to the healthcare industry can only be grounded with the conscious acknowledgment of the confidentiality concerns of patients. In this regard, this fragment discusses the different techniques that can be able to employ for privacy in healthcare such as Server Authentication, Triple DES. The incessant intrusion upon patients' confidentiality partly as a result of the compromise of their data stored in Cloud has attracted attention in the domain of big data in healthcare[10].

\section{Experimental Result}

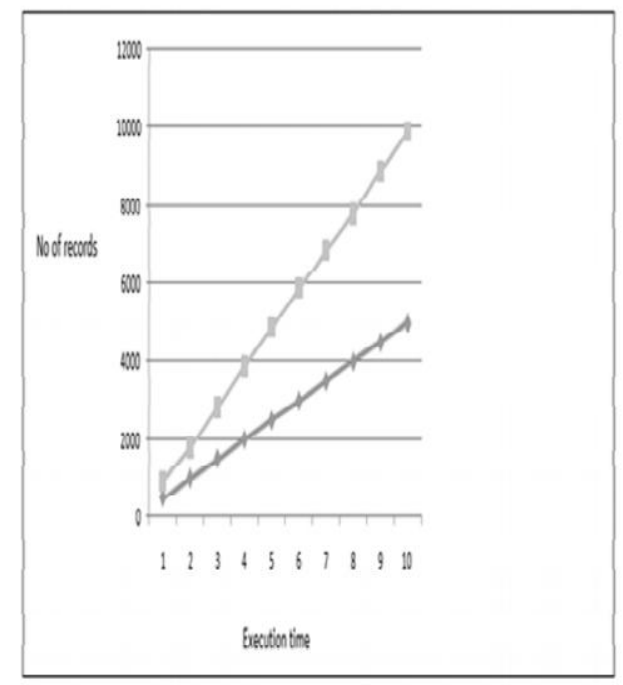

Fig. 3: Overall Execution Time

\section{Conclusion}

Based on above studies we are summarizing that there is very dangerous threats in big data related to secrecy of individual and it would cause him uncomfortable to sustain in life if any individual disorder is disclosed with surrounding peoples and may cause unsecure feeling in individuals mind.

So to avoid such a technological misshape there should be privacy preservation technique available in online system where user can authenticate or limit the use of confidentialdata by third party for example doctor, hospitals, Radiologist etc. By using proposed system we can ensure the person that the confidentialdata like 
name, address, religion etc will not be disclosed during the health monitoring activities.

\section{References}

[1] Kevin McCormack et al “ A Mathematical Solution to String Matching for Big Data Linking" Central Statistics Office, Cork, Ireland, IEEE Journal of Statistical Science and Application,IEEE (2017) P.P.39-55

[2] Gonzales, A Leigh, J Peisert, et al. "Monitoring Big Data Transfers Over International Research Network Connections", Lawrence Berkeley National Laboratory,IEEE (2017).

[3] Bing Niu et al "Analysis and Modeling for Big Data in Cancer Research",BioMed Research International, Hindawi,IEEE(2017).

[4] Stefano M. Iacus "Big Data and Social Science - A Practical Guide to Methods and Tools", University of Milan,IEEE JUNE2017,vol.78.

[5] HarshitKuma "Review paper on Big Data in healthcare informatics",International Research Journal of Engineering and Technology (IRJET) ,IEEE(2017),vol.04,P.P.197-201.

[6] P.Subhapriya et al" Healthcare Prediction Analysis in Big Data using Random Forest Classifier”, International Journal Of Advance Research Ideas And Innovations In Technology,IEEE(2017),vol.3,P.P.494-496.

[7] J.Archenaa "A Survey Of Big Data Analytics in Healthcare and Government", Research Scholar, MET University,Chennai,Science Direct(2015),P.P.408-413.

[8] DurgaAmarnath M. Budida et al "A Review Paper on Design and Implementation of Smart Health Care System using IoT", International Conference On Emanations in Modern Engineering Science and Management,IEEE(2017),vol.3,P.P.05-09.

[9] Lidong Wang et al "Big Data in Medical Applications and Health Care”, Department of Engineering Technology, Mississippi Valley State University, USA,Science Publication(2015)

[10] Javier Andreu-Perez et al " Big Data for Health",IEEE Journal Of Biomedical And Health Informaticas,IEEE JULY2015,vol.19,P.P1193-1208.

[11] ManojJayabalan et al "Exploring the Impact of Big Data in Healthcare and Techniques in Preserving Patients' Privacy", International Journal of Computer Science and Network Security, IEEE August-2017, vol.17,P.P.143-149.

[12] AshwinBelleet al "Big Data Analytics in Healthcare"BioMed Research International,Hindwai(2015),P.P.1-16.

[13] AhemadMousaet al " Security and Privacy Issues in Ehealthcare Systems: Towards Trusted Services", International Journal of Advanced Computer Science and Application,IEEE(2016),vol.4,P.P.229-236.

[14] MoeenHassanalieragh et al "Health Monitoring and Management Using Internet-of- Things (IOT) Sensing with Cloudbased Processing: Opportunities and Challenges", IEEE International Conference on Services Computing, IEEE(2015),P.P.285292.

[15] Prof.Hiralal.M.Patel et al "Security \& Privacy Issues of Big Data in IOT based Healthcare System using Cloud Computing", International Journal on Recent and Innovation Trends in Computing and Communication,IEEE JUNE-2017,vol.5,P.P.26-30.

[16] Bhoomika.B.K. et al "Secured Smart Healthcare Monitoring System Based on IoT", International Journal on Recent and Innovation Trends in Computing and Communication,IEEE JULY2015,vol.3,P.P.4958-4961.

[17] K. Natarajan et al "Smart Health Care System Using Internet of Things", Journal of Network Communications and Emerging Technologies, IEEE March-2016,vol.6,P.P.37-42.

[18] Dr. M.Umashankar "Privacy Preserving Healthcare System using secured cloud environment" in The International Journal Of Engineering And Computer vol. 6, no. 1, 2017

[19] Md. ZahidIbnaAlam "Security \& Privacy Issues of Big Data in IOT based Healthcare System using Cloud Computing" in the International Journal on Recent and Innovation Trends in Computing and Communication vol.6, no.1, 2017

[20] S.Sathya , DrT.Sethukarasi "EFFICIENT PRIVACY PRESER VATION TECHNIQUE FOR HEALTHCARE RECORDS USING BIG DATA" in the International Conference On Information Communication And Embedded System vol.23, no.1, 2016

[21] Husain Subedar et.al. "Privacy and Security Concerns for Health Data Collected Using Off-the-Shelf Health Monitoring Devices" in the e-Health Pervasive Wireless Applications and Services vol. 9 no. 1,2015

[22] S. Balaji et.al. "A Cloud based Big Data Based Online Health Analytics for Rural NICUs and PICUs in India: Opportunities and Challenges" in the 2017 IEEE 30th International Symposium vol.22,no1,2017

[23] TheodorosMavroeidakos "Centralized Management of Medical Big Data in Intensive Care Unit: A Security Analysis" IEEE JUNE2017, vol.5, P.P.26-30

[24] Md. ZahidIbnaAlam "Security \& Privacy Issues of Big Data in IOT based Healthcare System using Cloud Computing" in the International Journal on Recent and Innovation Trends in Computing and Communication vol.6, no.2, 2017

[25] S.Sathya , DrT.Sethukarasi "EFFICIENT PRIVACY PRESERVATION TECHNIQUE FOR HEALTHCARE RECORDS USING BIG DATA" in the International Conference On Information Communication And Embedded System vol.23, no.3, 2016

[26] S.Sathya , DrT.Sethukarasi "EFFICIENT PRIVACY PRESER VATION TECHNIQUE FOR HEALTHCARE RECORDS USING BIG DATA" in the International Conference On Information Communication And Embedded System vol.23, no.4, 2016

[27] Justice Asare-Frempong "Exploring the Impact of Big Data in Healthcare and Techniques in Preserving Patients' Privacy" in the IJCSNS International Journal of Computer Science and Network Security vol.17, no.8, 2017 